\title{
CIENCIAMATRIA
}

Revista Interdisciplinaria de Humanidades, Educación, Ciencia y Tecnología

Año VII. Vol. VII. N¹3. Julio - Diciembre. 2021

Hecho el depósito de ley: pp201602FA4721

ISSN-L: 2542-3029; ISSN: 2610-802X

Universidad Nacional Experimental Francisco de Miranda (UNEFM). Santa Ana de Coro. Venezuela

Marcos Adrián Luna-Rojas; Carlos Marcelo Ávila-Mediavilla

DOI $10.35381 / \mathrm{cm} . v 7 i 13.476$

\section{Motivación y Educación Física en la Virtualidad}

Motivation and Physical Education in Virtuality

\author{
Marcos Adrián Luna-Rojas \\ marcos.luna.66@est.ucacu.edu.ec \\ Universidad Católica de Cuenca, Azogues \\ Ecuador \\ https://orcid.org/0000-0002-7939-3673 \\ Carlos Marcelo Ávila-Mediavilla \\ cavilam@ucacue.edu.ec \\ Universidad Católica de Cuenca, Cuenca \\ Ecuador \\ https://orcid.org/0000-0002-2649-9634
}

Recibido: 10 de marzo de 2021

Aprobado: 15 de junio de 2021 


\author{
CIENCIAMATRIA \\ Revista Interdisciplinaria de Humanidades, Educación, Ciencia y Tecnología \\ Año VII. Vol. VII. N¹3. Julio - Diciembre. 2021 \\ Hecho el depósito de ley: pp201602FA4721 \\ ISSN-L: 2542-3029; ISSN: 2610-802X \\ Universidad Nacional Experimental Francisco de Miranda (UNEFM). Santa Ana de Coro. Venezuela
}

Marcos Adrián Luna-Rojas; Carlos Marcelo Ávila-Mediavilla

\title{
RESUMEN
}

Durante la pandemia de COVID-19, existen muchos desafíos, a nivel nacional y mundial, incluidos el empleo, la movilidad, los deportes y la educación, siendo necesario prevenir la propagación del virus. Por lo tanto, muchos niños difícilmente pueden seguir estudiando y practicando debido a la falta de Internet o dispositivos electrónicos que aseguren el desarrollo de contenidos en cada nivel escolar. También con esta situación perdieron el interés. Por tal razón también afectó en el ámbito de la educación física, por ello en el estudio realizado y los resultados obtenidos los padres de familia consideran de gran importancia motivarlos a aprender todo lo que la escolarización aporta a su formación. Es decir que la motivación es probablemente el eje principal del plan de aprendizaje, por lo que siempre debe estar presente en cada momento de la clase.

Descriptores: Tecnología educacional; programa informático didáctico; enseñanza asistida por ordenador. (Palabras tomadas del Tesauro UNESCO).

\begin{abstract}
During the COVID-19 pandemic, there are many challenges, nationally and globally, including employment, mobility, sports, and education, making it necessary to prevent the spread of the virus. Therefore, many children can hardly continue studying and practicing due to the lack of Internet or electronic devices that ensure the development of content at each school level. Also with this situation they lost interest. For this reason, it also affected the field of physical education, which is why in the study carried out and the results obtained, parents consider it very important to motivate them to learn everything that schooling contributes to their training. In other words, motivation is probably the main axis of the learning plan, so it must always be present at every moment of the class.
\end{abstract}

Descriptors: Educational technology; educational software; computer assisted instruction. (Words taken from the UNESCO Thesaurus). 


\section{CIENCIAMATRIA \\ Revista Interdisciplinaria de Humanidades, Educación, Ciencia y Tecnología \\ Año VII. Vol. VII. N`13. Julio - Diciembre. 2021 \\ Hecho el depósito de ley: pp201602FA4721 \\ ISSN-L: 2542-3029; ISSN: 2610-802X \\ Universidad Nacional Experimental Francisco de Miranda (UNEFM). Santa Ana de Coro. Venezuela}

Marcos Adrián Luna-Rojas; Carlos Marcelo Ávila-Mediavilla

\section{INTRODUCCIÓN}

La pandemia del covid-19 por la que está atravesando actualmente todo el mundo, ha ocasionado crisis de todos los aspectos, en cuanto a la educación la emergencia provocó el cierre total de las clases presenciales en más de 190 países, con la finalidad de que el virus no se propague y así disminuir su impacto de forma global. La Organización de las Naciones Unidas para la Educación, la Ciencia y la Cultura (UNESCO) indica que la educación que se llevaba a cabo de manera presencial, se realizará de forma virtual en todas las instituciones en todos sus niveles, con el fin de cuidar la integridad de todos los estudiantes y sus familias (CEPAL-UNESCO, 2020).

Así mismo, Vialart (2020) menciona que, ya existía un deterioro en la situación social, inclusive antes de que se presente la pandemia, es por ello por lo que la crisis afecta a la sociedad tanto en la salud como en la educación provocando una falta de empleo lo que genera que la pobreza aumente significativamente. Por otra parte, la educación ha generado tres ámbitos que serán usados para la nueva modalidad de estudio y teletrabajo enfocados en las distintas estrategias que serán aplicados en la clase virtual, para que los alumnos mantengan un buen aprendizaje. Además, el internet ofrece diversos servicios, medios y recursos para que exista un correcto proceso de enseñanzaaprendizaje.

Así también, la motivación es una herramienta fundamental para los docentes y que el aprendizaje sea el correcto ayudando a que los alumnos puedan obtener resultados positivos, es por ello que se han realizado varias investigaciones en cuanto a la motivación y el efecto que tendrá en el estudiantado, así mismo se evidencian las consecuencias positivas tanto como negativas que se obtendrán en los mismos, siendo evidencia las distintas actividades que se presenta en el día a día en la institución en la que se encuentren los individuos (Carabelli, 2020).

Con ésta investigación se pretende conocer de qué manera la motivación puede aportar a la enseñanza de educación física de forma virtual en el nivel medio de educación general básica de la Unidad Educativa "Joaquín Fernández de Córdova". 


\section{CIENCIAMATRIA \\ Revista Interdisciplinaria de Humanidades, Educación, Ciencia y Tecnología \\ Año VII. Vol. VII. N`13. Julio - Diciembre. 2021 \\ Hecho el depósito de ley: pp201602FA4721 \\ ISSN-L: 2542-3029; ISSN: 2610-802X \\ Universidad Nacional Experimental Francisco de Miranda (UNEFM). Santa Ana de Coro. Venezuela}

Marcos Adrián Luna-Rojas; Carlos Marcelo Ávila-Mediavilla

\section{Referencial teórico}

\section{Motivación y su importancia}

Durante la historia, en el año de 1920 - 1960 la motivación se asocia con una investigación experimental misma que se asemeja a las conductas motoras del individuo, instinto e impulsos. Esta fase busca que el individuo restaure el estado de equilibrio en relación con los factores que determinan la motivación, como lo son los refuerzos. Pasados los años de 1960, surgieron teorías cognitivas sobre la motivación que se basan en la experiencia consciente, relacionado con interés a la motivación de rendimiento y la importancia de los logros personales de un individuo (Naranjo Pereira, 2009).

Durante años expertos han estudiado la motivación y han expresado que, la psicología motivacional forma parte de una serie de términos y principios fundamentales en la plantación de dichas teorías. Un buen conocimiento sobre estos aspectos es esencial para que el docente mantenga la motivación activa en los estudiantes. De la misma forma, es necesario tener en cuenta las circunstancias, la relevancia y el valor en las que se desarrolló, ya que la motivación ha estado presente durante mucho tiempo en la historia del hombre y su evolución (Jacovkis, 2011).

Según Palmero et al. (2008), expone que la motivación y sus teorías han sido fundamentadas en ideologías clásicas relacionadas con la filosofía. Así también, se relaciona con las teorías psicológicas, que alcanzan a ser relevantes en la época. Por ello, es que los distintos enfoques de la psicología a la motivación se consideran que pueden ser basados en aspectos como la biología y la conducta, mismos que permitirán el avance y la evolución cronológica de orientaciones en motivación. De acuerdo con ello, se puede identificar los puntos clave de la motivación para el desarrollo de la teoría motivacional y su influencia en las distintas situaciones.

En la motivación intervienen procesos afectivos, emocionales y sentimentales, así como también tendencias y procesos cognitivos fundamentales del ser humano. Para la pedagogía psicológica, es importante la motivación en el estudio y aprendizaje, puesto que motivar a los estudiantes es estimular el deseo de conocer, investigar y despertar de 


\section{CIENCIAMATRIA \\ Revista Interdisciplinaria de Humanidades, Educación, Ciencia y Tecnología \\ Año VII. Vol. VII. NN13. Julio - Diciembre. 2021 \\ Hecho el depósito de ley: pp201602FA4721 \\ ISSN-L: 2542-3029; ISSN: 2610-802X \\ Universidad Nacional Experimental Francisco de Miranda (UNEFM). Santa Ana de Coro. Venezuela}

Marcos Adrián Luna-Rojas; Carlos Marcelo Ávila-Mediavilla

la curiosidad de aquello, que no es de su conocimiento. Es por ello, que la motivación estudiantil es fundamental al momento de profundizar en las características específicas del estudio como base de este. Por lo tanto, los docentes han referido que los malos resultados se relacionan con una mala motivación y posterior a ello se presentan anomalías en la enseñanza-aprendizaje (Morera-méndez, 1995).

\section{Tecnología y Educación}

Según Mesa (2012), postula que, desde hace años atrás, la tecnología ha comenzado a estar presente en la vida de las personas, misma que ha ido adoptando distintas posturas a medida que pasa el tiempo, es en ese entonces que en el siglo XXI se le reconoce por primera vez como las Tecnología de la Información y de la Comunicación como una herramienta utilizada para la comunicación entre las personas. Estas han ido evolucionando día a día pues siempre está demostrando nuevos avances para la comunicación, así como también mejoras en la innovación, digitalización, elevada calidad de imagen, interconexión mejorada, entre otras.

La creación de estas herramientas, según Diaz (2008) menciona que ha ayudado a la evolución de la tecnología en todo el mundo, pues por este medio se ha podido obtener métodos de estudios e investigación necesarias o complementarias para el aprendizaje de las personas. Es por ello, que su incorporación promete ayudar a la innovación de la educación e integración de nuevas estrategias metodológicas para el estudio, que es aquello que mejorara la calidad del estudiante y los conocimientos, siendo más valiosos para los futuros estudiantes que adquirirán los conocimientos, es decir, busca innovar a la educación.

La educación combinada con la tecnología es un invento, misma que pretende desarrollar un modelo enfocado en pensar y el que hacer desde el preescolar hasta la educación básica que ayudara al desarrollo de la capacidad de la resolución de problemas. Ramírez, Escalante y León (2008), mencionan que en Argentina y Chile fueron uno de los primeros países de Latinoamérica en implementar la educación con la tecnología, como base 


\section{CIENCIAMATRIA \\ Revista Interdisciplinaria de Humanidades, Educación, Ciencia y Tecnología \\ Año VII. Vol. VII. NN13. Julio - Diciembre. 2021 \\ Hecho el depósito de ley: pp201602FA4721 \\ ISSN-L: 2542-3029; ISSN: 2610-802X \\ Universidad Nacional Experimental Francisco de Miranda (UNEFM). Santa Ana de Coro. Venezuela}

Marcos Adrián Luna-Rojas; Carlos Marcelo Ávila-Mediavilla

fundamental para el desarrollo del enseñanza-aprendizaje, sin embargo, antes de dicha incorporación se realizó estudios del contexto, experiencia y trayectoria de la pedagogía, pero su principal objetivo es ofrecer una formación adecuada de los estudiantes.

Por lo tanto, la incorporación de la tecnología en la educación a medida que pasa el tiempo ha sufrido cambios que permiten una innovación, ya sea en la educación a distancia o la educación presencial, el impacto de los TIC proporciona nuevos paradigmas que prevalecen en la educación en la enseñanza-aprendizaje o evaluación. Además, se menciona que la educación en el futuro mostrara un incremento significativo en todo el mundo en todos sus ámbitos. Sin embargo, los países más desarrollados serán los primeros en tener acceso a la nueva educación (Díaz, 2008).

A partir de las transformaciones de la educación e incorporación de nuevas herramientas en todos los ámbitos educativos que va desde el preescolar hasta la educación superior, aun así, la tecnología también ha sufrido críticas sobre la eficiencia y eficacia de su rendimiento. Según Cano (1970) postula que la educación se enfrenta a desafíos al momento de hacer uso de la tecnología puesto que el docente debe contar con la adecuada formación sobre su implementación al momento del proceso de enseñanzaaprendizaje, para que los estudiantes adquieran conocimientos estables y que en un futuro sean exitosos y competitivos en su vida profesional.

Por lo tanto, la educación es de suma importancia para la evolución de un país. Los docentes necesariamente deben tener experiencia en utilizar estrategias para una adecuada implementación de conocimientos, sin embargo, algunos docentes que no cuentan con estos conocimientos pueden provocar dificultades del aprendizaje en sus estudiantes. Es por ello, que constantemente los docentes deben estar sujetos a capacitaciones que los ayuden a seguir innovando sus metodologías de enseñanzaaprendizaje, de manera que los estudiantes cada vez comprendan mejor los nuevos conocimientos (Mendoza Juárez \& Mamani Gamarra, 2012).

Los cambios en la educación que se han dado a medida que pasa los años, han sido necesarios para el desarrollo de país, así también, ayudan a la educación de las personas 


\section{CIENCIAMATRIA \\ Revista Interdisciplinaria de Humanidades, Educación, Ciencia y Tecnología \\ Año VII. Vol. VII. N¹3. Julio - Diciembre. 2021 \\ Hecho el depósito de ley: pp201602FA4721 \\ ISSN-L: 2542-3029; ISSN: 2610-802X \\ Universidad Nacional Experimental Francisco de Miranda (UNEFM). Santa Ana de Coro. Venezuela}

Marcos Adrián Luna-Rojas; Carlos Marcelo Ávila-Mediavilla

y apoyan a generar nuevos procesos de aprendizajes, mediante la educación es posible que se de soluciones a las distintas necesidades de formación de la sociedad, como puede ser de personas con tengan problemas en el manejo de la educación por el medio virtual, por lo que es importante la nueva modalidad de estudio en la educación actual (Castillo et al., 2017).

La motivación es aquello que ayuda a generar una conducta en el ser humano, mismo que se enlaza con las necesidades del individuo y le incita a realizar una acción ya sea de origen físico o psicológico. Al aparecer una necesidad, esta entra en un estado de tensión, insatisfacción o inconformismo que es lo que necesariamente se requiere para que se genere una acción y posterior al mismo una reacción que es aquello que va a solucionar dicha necesidad y así volver a sentir tranquilidad (Castillo et al., 2017).

Según Tirado, Santos y Tejero (2013) exponen que, la motivación es fundamental en la educación para favorecer el aprendizaje de los estudiantes pues una vez interesados y motivados por adquirir nuevos conocimientos los estudiantes valoraran lo aprendido y almacenaran dicha información vinculando con la experiencia previa. Es por ello, que la importancia de los métodos de enseñanza es fundamental para que los estudiantes le den un buen uso, utilidad al tema tratado y el sentido del contenido, mismos que se consiguen al principio del curso.

\section{Motivación y Educación Física}

La motivación intrínseca es un elemento fundamental para el docente al momento de comenzar con su clase, Aguilar, Gonzales y Aguilar (2016) menciona que está enfocada a que exista una participación constante de los alumnos en relación a una actividad que sea de interés, cause placer y satisfacción al momento de realizar la actividad. Por lo tanto, cuando los alumnos experimentan dichas emociones se incrementará su interés por conocer y en la asignatura de educación física ayudará mejorando la calidad de aprendizaje del estudiante. 


\section{CIENCIAMATRIA \\ Revista Interdisciplinaria de Humanidades, Educación, Ciencia y Tecnología \\ Año VII. Vol. VII. NN13. Julio - Diciembre. 2021 \\ Hecho el depósito de ley: pp201602FA4721 \\ ISSN-L: 2542-3029; ISSN: 2610-802X \\ Universidad Nacional Experimental Francisco de Miranda (UNEFM). Santa Ana de Coro. Venezuela}

Marcos Adrián Luna-Rojas; Carlos Marcelo Ávila-Mediavilla

Se han generado nuevos métodos de estudio por razones ajenas a los docentes, se hace referencia las TIC como herramienta pedagógica, misma que por sus avances ha sido necesario desarrollar técnicas, métodos y estrategias para la enseñanza de los estudiantes, empezando por la adaptación a la nueva modalidad de estudio procurando que los conocimientos sean asimilados y procesados correctamente. Los docentes requieren de un adecuado conocimiento de las TIC para que no existan dificultades en el aprendizaje de los estudiantes y los conocimientos sean manejados de manera fluida, mas no dando paso al desvió de la concentración y al conocimiento negativo o desinformación (Peña Rodríguez \& Otálora Porras, 2018).

Es fundamental tener claro que la enseñanza y el aprendizaje de una persona esta enlazado directamente con las herramientas que se utilicen por el docente, de tal forma, que se ajusten a las necesidades del grupo de estudiantes al que le está impartiendo sus conocimientos Torres y Cobo (2017), postulan que la importancia de la participación activa del estudiantado, es mantener la motivación e interés en un rango elevado sobre el tema que se desarrollará, todo esto para obtener resultados positivos, de manera que los conocimientos impartidos estén presentes de manera significativa. Es por ello, que el docente debe conocer las características y necesidades de cada uno de ellos.

La pedagogía es un elemento muy importante en la educación virtual ya que no se enfoca únicamente en la tecnología, pero que esta juega un papel muy importante en la edificación de conocimientos solidos de los estudiantes, por lo tanto, diversos estudios se han focalizado en el desarrollo de estrategias para que el docente adopte un correcto método la enseñanza dirigidas al proceso de enseñanza-aprendizaje, mismas que ayudaran a que exista la participación activa de los estudiantes, sin embargo, se debe considerar al entorno social, cultural y tecnológico como elementos del sistema educativo (Martínez et al., 2018).

Según Delgado y Solano (2012), postulan que, en la enseñanza virtual es necesario contar con herramientas que faciliten el acceso a las distintas actividades establecidas por los docentes, mismas que ayudaran a los estudiantes a participar en la clase y 


\section{CIENCIAMATRIA \\ Revista Interdisciplinaria de Humanidades, Educación, Ciencia y Tecnología \\ Año VII. Vol. VII. N¹3. Julio - Diciembre. 2021 \\ Hecho el depósito de ley: pp201602FA4721 \\ ISSN-L: 2542-3029; ISSN: 2610-802X \\ Universidad Nacional Experimental Francisco de Miranda (UNEFM). Santa Ana de Coro. Venezuela}

Marcos Adrián Luna-Rojas; Carlos Marcelo Ávila-Mediavilla

posterior a ello y mediante ella construyan conocimientos significativos, cooperen e interaccionen con los demás participantes que están haciendo uso de la misma. Una de estas herramientas es la plataforma Moodle que consta de un sistema libre en la gestión de los cursos, por medio de este el docente puede crear nuevos espacios de aprendizaje que se llevan en línea que consta de distintas actividades que deberán ser desarrolladas por los estudiantes.

Existen distintas estrategias, entre ellas están las estrategias didácticas para la enseñanza-aprendizaje de los estudiantes, por lo tanto, es importante definir cada una de ellas. Las estrategias para el aprendizaje se enfocan en las habilidades que el individuo posee y la forma que lo utiliza al momento de aprender y dar soluciones a las distintas situaciones en su vida diaria, teniendo en cuenta la experiencia ya adquirida. Mientras que las estrategias de enseñanza son aquellas que son establecidas por el docente que es quien brinda la información adecuada sobre distintos temas expuestos, y el estudiante es aquel que se encarga de procesarla y almacenarla con la demás información (Arteaga, C; Enríquez, N y Chuquimia, 1967).

Existen varios tipos de estrategias que están conformadas por diversas técnicas de enseñanza, una de ellas son las técnicas centradas en la individualización de la enseñanza que prioriza en adaptar las necesidades e intereses de los estudiantes. Las herramientas que se utilicen facilitaran una mejora de la autonomía, control en el ritmo de enseñanza y encadenamientos para el aprendizaje, para la aplicación de dicha técnica es necesario que el docente posea una buena relación con el estudiante y que de esta forma se designe actividades de auto realización y de distintos niveles de dificultad (Betancur Chicué et al., 2015).

Así también, las técnicas de exposición y participación en grupo ayudarán a la construcción de nuevos conocimientos a partir de la información brindada anteriormente. También se manifiesta dos roles para el desarrollo de las actividades, el primero es del expositor que puede ser el docente, un experto o un estudiante, con el conocimiento suficiente sobre el tema y el segundo es el grupo que recepta la información, Pando 


\section{CIENCIAMATRIA \\ Revista Interdisciplinaria de Humanidades, Educación, Ciencia y Tecnología \\ Año VII. Vol. VII. N¹3. Julio - Diciembre. 2021 \\ Hecho el depósito de ley: pp201602FA4721 \\ ISSN-L: 2542-3029; ISSN: 2610-802X \\ Universidad Nacional Experimental Francisco de Miranda (UNEFM). Santa Ana de Coro. Venezuela}

Marcos Adrián Luna-Rojas; Carlos Marcelo Ávila-Mediavilla

(2018), mismo que será responsable de las actividades que después deberá compartir con todo el grupo de trabajo.

Por otra parte, se habla de las técnicas del trabajo colaborativo que es todo lo opuesto a la técnica anterior, esta intenta edificar conocimientos de forma grupal para que las estructuras ayuden a la comunicación en el grupo. Los resultados obtenidos deberán ser expuestos en el grupo, se necesita que todos participen y cooperen de forma explícita sobre la información y dando paso a que exista intercambio de ideas entre los integrantes. Así mismo, el docente antes de que se comience con el trabajo, deberá dar las debidas normas para la estructuración de la actividad y mantener un seguimiento de cerca (Sánchez, O; Collazos, C y Jiménez, 2018).

\section{METODOLOGÍA}

El diseño de investigación responde a un tipo descriptivo de corte transversal con un enfoque descriptivo - analítico - inductivo para el desarrollo de la investigación, en dónde nos permitirá considerar las variables para el desarrollo de la investigación.

En el presente tema de investigación de motivación y Educación Física en enseñanza virtual como base para la educación primaria, consiste en una indagación de carácter social por el desconocimiento y la importancia que tienen la motivación en la práctica de Educación Física de las personas, el mejoramiento que beneficiará a los niños/as del plantel en el subnivel medio.

La población se realizó a los padres de familia de los niños del nivel medio quinto, sexto y séptimo de Educación General Básica, de la Unidad Educativa "Joaquín Fernández de Córdova" de la ciudad de Cuenca, en un total de N.-90 padres de familia, la muestra fue N.-74 encuestados. Las encuestas se realizaron a través de la aplicación de forms.office.com, lo que nos permitió valorar la variable independiente Motivación y Educación Virtual y la variable dependiente Educación Física, se valorara a través del programa Excel en donde serán insertados todos los datos obtenidos para su posterior análisis estadístico utilizando el paquete SPSS los mismos que ayudarán a una exhibición 


\section{CIENCIAMATRIA}

Revista Interdisciplinaria de Humanidades, Educación, Ciencia y Tecnología

Año VII. Vol. VII. №13. Julio - Diciembre. 2021

Hecho el depósito de ley: pp201602FA4721

ISSN-L: 2542-3029; ISSN: 2610-802X

Universidad Nacional Experimental Francisco de Miranda (UNEFM). Santa Ana de Coro. Venezuela

Marcos Adrián Luna-Rojas; Carlos Marcelo Ávila-Mediavilla

real y confiable de los resultados. El instrumento fue de investigación fue validado por el Alpha de Cronbatch con un valor de 0.750

\section{RESULTADOS}

En el proceso de enseñanza-aprendizaje están involucrados varios actores como son: estudiantes, docentes, padres de familia y los contenidos que se impartirán durante los diferentes periodos en la etapa escolar, para esto se aplicó una encuesta en donde están presentes las variables de la investigación, a continuación está el detalle de los resultados obtenidos luego de aplicar el instrumento a los padres de familia de quinto, sexto y séptimo año de educación general básica de la Unidad Educativa "Joaquín Fernández de Córdova" 2020-2021 Cuenca - Ecuador.

Cuando se realizó el análisis de normalidad se determinó que todas las variables son paramétricas.

A continuación se presentan los resultados más relevantes que permiten responder el objetivo de investigación.

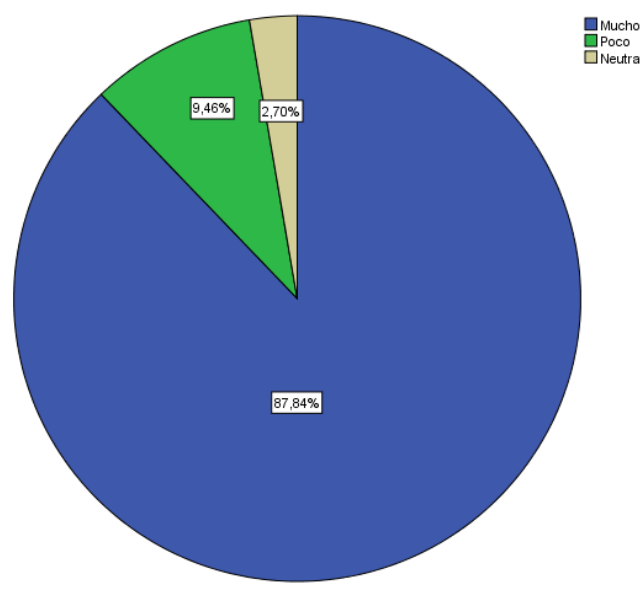

Figura 1. Motivación en clases.

Fuente: Encuesta. 


\section{CIENCIAMATRIA}

Revista Interdisciplinaria de Humanidades, Educación, Ciencia y Tecnología

Año VII. Vol. VII. №13. Julio - Diciembre. 2021

Hecho el depósito de ley: pp201602FA4721

ISSN-L: 2542-3029; ISSN: 2610-802X

Universidad Nacional Experimental Francisco de Miranda (UNEFM). Santa Ana de Coro. Venezuela

Marcos Adrián Luna-Rojas; Carlos Marcelo Ávila-Mediavilla

Un porcentaje muy elevado de padres de familia, indica que el docente motiva a los estudiantes al inicio de cada clase, el resultado es el siguiente: Mucho 87,84\%, Poco $9,46 \%$ y Neutral el 2,70\% por lo expuesto la motivación es muy utilizada en el desarrollo de las clases, datos que demuestran la gran importancia en el desarrollo de las clases.

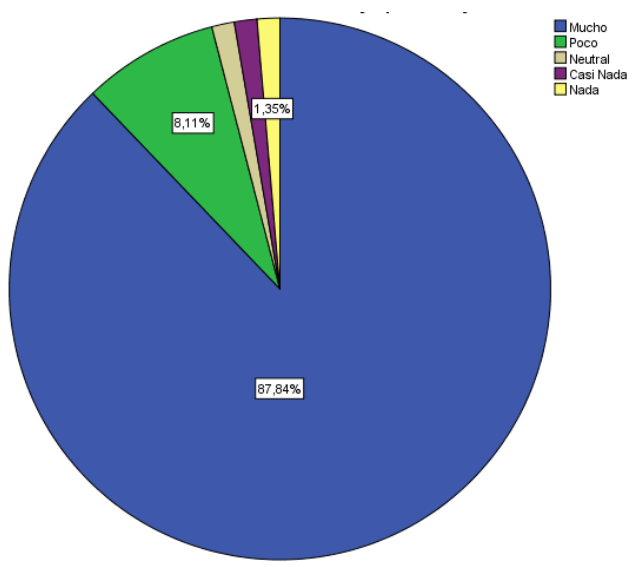

Figura 2. Motivación y aprendizaje.

Fuente: Encuesta.

Los padres de familia consideran que la motivación es vital para el aprendizaje de los estudiantes, el resultado es el siguiente: Mucho $87,84 \%$, Poco $8,11 \%$ y Neutral, Casi Nada y Nada el 1,35\%. Se puede evidenciar a gran escala que la motivación es la parte fundamental para el aprendizaje de los estudiantes. 


\section{CIENCIAMATRIA}

Revista Interdisciplinaria de Humanidades, Educación, Ciencia y Tecnología

Año VII. Vol. VII. №13. Julio - Diciembre. 2021

Hecho el depósito de ley: pp201602FA4721

ISSN-L: 2542-3029; ISSN: 2610-802X

Universidad Nacional Experimental Francisco de Miranda (UNEFM). Santa Ana de Coro. Venezuela

Marcos Adrián Luna-Rojas; Carlos Marcelo Ávila-Mediavilla

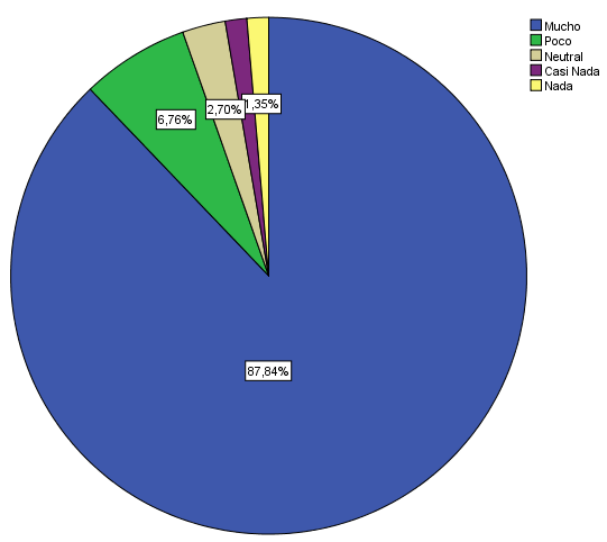

Figura 3. Estrategias de enseñanza-aprendizaje.

Fuente: Encuesta.

Los padres de familia, consideran que el docente debe contar con estrategias adecuadas para la enseñanza-aprendizaje con los siguientes resultados: Mucho 87,84\%, Poco $6,76 \%$, Neutral $2,70 \%$, Casi nada y Nada $1,35 \%$. Se puede evidenciar que el mayor porcentaje está en la categoría Mucho, siendo un factor positivo para un desarrollo óptimo en la enseñanza-aprendizaje.

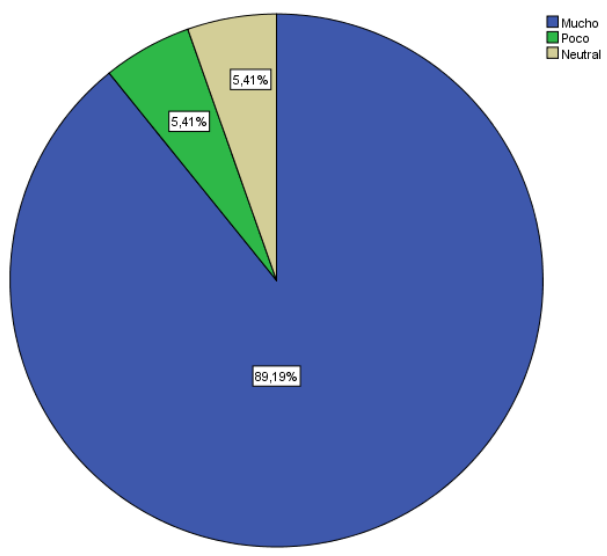

Figura 4. Herramientas en Educación Virtual

Fuente: Encuesta. 


\section{CIENCIAMATRIA}

Revista Interdisciplinaria de Humanidades, Educación, Ciencia y Tecnología

Año VII. Vol. VII. №13. Julio - Diciembre. 2021

Hecho el depósito de ley: pp201602FA4721

ISSN-L: 2542-3029; ISSN: 2610-802X

Universidad Nacional Experimental Francisco de Miranda (UNEFM). Santa Ana de Coro. Venezuela

Marcos Adrián Luna-Rojas; Carlos Marcelo Ávila-Mediavilla

Los padres de familia manifiestan que es necesario que el docente conozca las herramientas utilizadas en educación virtual, el resultado es el siguiente: Mucho 89,19\% y Poco 5,41\% y Neutral 5,41\%. Evidentemente la supremacía en el porcentaje a favor de que es necesario que el docente conozca las herramientas utilizadas en educación virtual, por lo que se les debe dar la importancia necesaria a la formación profesional.

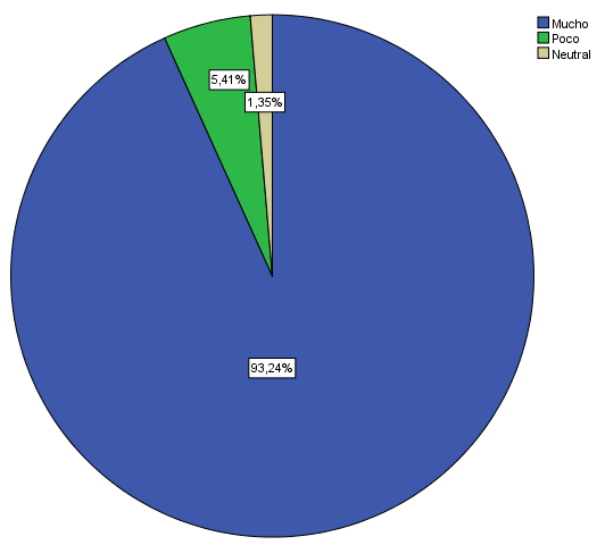

Figura 5. Preparación Docente.

Fuente: Encuesta.

Los padres de familia, manifiestan que el docente debe contar con suficientes conocimientos antes de impartirlos en clase, el resultado es el siguiente: Mucho 93,24\%, Poco $5,41 \%$ y Neutral $1,35 \%$. Un alto porcentaje consideran mucho, dando a entender que el docente debe contar con suficientes conocimientos antes de impartirlos en clase siendo estos claves, para una posterior transmición adecuada de los mismos.. 


\section{CIENCIAMATRIA \\ Revista Interdisciplinaria de Humanidades, Educación, Ciencia y Tecnología \\ Año VII. Vol. VII. N¹3. Julio - Diciembre. 2021 \\ Hecho el depósito de ley: pp201602FA4721 \\ ISSN-L: 2542-3029; ISSN: 2610-802X \\ Universidad Nacional Experimental Francisco de Miranda (UNEFM). Santa Ana de Coro. Venezuela}

Marcos Adrián Luna-Rojas; Carlos Marcelo Ávila-Mediavilla

\section{PROPUESTA}

En este sentido, los conocimientos y estrategias que un docente tenga sobre la motivación en las aulas son de gran importancia en su práctica didáctica y pedagógica. Pero si deseamos que los docentes utilicen la motivación en el aprendizaje de forma eficiente, considero que primero se debe conocer cómo se la utiliza de manera efectiva en el contexto escolar, es decir, desarrollar la motivación por aprender y no solo la motivación para realizar una tarea.

En tal virtud lo importante sería desarrollar una propuesta de ejecución de estrategias de aprendizaje que aporten a la motivación del aprendizaje de los estudiantes. Esta propuesta si bien es cierto no es ni será la definitiva trata de dar la importancia a la aplicación óptima de la motivación en el proceso de enseñanza aprendizaje en la asignatura de Educación Física.

En la intención de aportar a la formación de los docentes, se presenta los siguientes puntos que pueden ser considerados importantes para el desarrollo de las clases de Educación Física:

1.- No es suficiente que el docente motive a sus estudiantes, sería recomendable que su planificación tenga varios elementos que aporten a alcanzar esta finalidad.

2.- Siempre tiene que estar presente la motivación en el desarrollo de los aprendizajes, puesto que esto ayudará a que los estudiantes se activen cognitivamente y aprendan de mejor manera.

3.- No solo se debería tener conocimientos de motivación, ya que sería más importante aplicarlos adecuadamente para incentivar a los estudiantes y que mantengan el interés por descubrir nuevos aprendizajes.

Para el presente plan Estratégico Didáctico y de Motivación para las Clase de Educación Física se ha tomado como referencia las siguientes competencias:

-Proporcionar suficiente tiempo. 3-4 tareas por sesión. Mínimo 8-10 sesiones por unidad didáctica. 


\section{CIENCIAMATRIA \\ Revista Interdisciplinaria de Humanidades, Educación, Ciencia y Tecnología \\ Año VII. Vol. VII. N¹3. Julio - Diciembre. 2021 \\ Hecho el depósito de ley: pp201602FA4721 \\ ISSN-L: 2542-3029; ISSN: 2610-802X \\ Universidad Nacional Experimental Francisco de Miranda (UNEFM). Santa Ana de Coro. Venezuela}

Marcos Adrián Luna-Rojas; Carlos Marcelo Ávila-Mediavilla

-Actividades novedosas. BodyPump, BodyCombat, CrossFit, Kinball, Zumba, Raids de aventura, barranquismo, TIC: Endomondo, Facebook.

- Reglas para que todos sean partícipes. Tocar todos los balones.

- Importancia del esfuerzo y la superación personal: Evaluación significativa y continua del proceso.

- Reconocer el progreso individual. No sólo hay que dar feedback cuando la ejecución es incorrecta.

- Feedback positivo. Feedback prescriptivo, interrogativo y afectivo. Tipo sándwich.

- Utilizar la música. Trabajando la resistencia a través de la carrera, juegos deportivos, expresión corporal.

- Dar posibilidad de elección. Objetivos, contenidos, reglas, espacio, tiempo, material, técnica de enseñanza, normas, evaluación.

- Explicar los objetivos didácticos. En cada tarea, abdominales por salud y estabilidad del tronco.

- Estilos participativos. Enseñanza recíproca, grupos reducidos, microenseñanza: Dirigir calentamiento o sesión.

- Fomentar la interacción. Estilo socializador: Objetivo común, todos necesarios, cambios de grupo (baraja española).

- Evitar rivalidad y desarrollar autocontrol. Utilizar el símil de la lata de coca cola para prevenir problemas entre los estudiantes.

- Clima de preocupación e inteligencia emocional. Interesarse por la vida del estudiante, tratar por el nombre, percibir y transmitir emociones

\section{CONCLUSIONES}

La motivación en Educación Física en edades tempranas ayuda a que los niños/as mejoren su interés en cada una de las actividades que se le presenten a lo largo de su vida estudiantil, deportiva e incluso de la vida cotidiana. 


\section{CIENCIAMATRIA \\ Revista Interdisciplinaria de Humanidades, Educación, Ciencia y Tecnología \\ Año VII. Vol. VII. N¹3. Julio - Diciembre. 2021 \\ Hecho el depósito de ley: pp201602FA4721 \\ ISSN-L: 2542-3029; ISSN: 2610-802X \\ Universidad Nacional Experimental Francisco de Miranda (UNEFM). Santa Ana de Coro. Venezuela}

Marcos Adrián Luna-Rojas; Carlos Marcelo Ávila-Mediavilla

Durante el proceso de investigación y también basado en la experiencia, se puede considerar que es un factor de gran importancia para que los estudiantes adquieran atracción por aprender la asignatura y poder realizar toda actividad recreativa o física.

En distintas situaciones se ha podido observar la falta de interés por trabajar algunos ejercicios, sin embargo, con la ayuda de motivación se puede lograr que se integren a las actividades propuestas por el docente.

Existen estudiantes que no tienen dificultad para realizar los diferentes ejercicios, pero también hay otros que pierden interés por ejecutarlos, ante esto podemos decir que pueden intervenir varios factores entre ellos están una planificación mal estructurada, falta de motivación por parte del docente del área, poco interés en las actividades por parte de los estudiantes en las clases virtuales, etc., sin embargo ante todas estas probabilidades a los menores se les dificulta trabajar de manera sistemática cada una de las actividades propuestas en el área de Educación Física.

El desconocimiento de la importancia que tiene el realizar actividad física en los niños/as del subnivel medio incentivaron a investigar este tema en donde se pudo conocer que la motivación en educación virtual aportará de mamera significativamente en el desarrollo de la actividad física obteniendo resultados satisfactorios hacia posibles nuevos talentos deportivos, ya que los deportistas nacen y también se hacen.

\section{REFERENCIAS}

Aguilar, J., González, D., \& Aguilar, A. (2016). Un modelo estructural de motivación intrínseca [A structural model of intrinsic motivation]. Acta de Investigación Psicológica, 6(3), 2552-2557. https://doi.org/10.1016/j.aipprr.2016.11.007

Arteaga, C; Enríquez, N y Chuquimia, L. (2015). [Methodological challenges in virtual education Approach to the complexities of virtual education and the rescue of the value of social contact]. Angewandte Chemie International Edition, 6(11), 951952., 10, 99-114. https://n9.cl/3jql9 


\section{CIENCIAMATRIA}

Revista Interdisciplinaria de Humanidades, Educación, Ciencia y Tecnología

Año VII. Vol. VII. N¹3. Julio - Diciembre. 2021

Hecho el depósito de ley: pp201602FA4721

ISSN-L: 2542-3029; ISSN: 2610-802X

Universidad Nacional Experimental Francisco de Miranda (UNEFM). Santa Ana de Coro. Venezuela

Marcos Adrián Luna-Rojas; Carlos Marcelo Ávila-Mediavilla

Betancur Chicué, V., Cárdenas Rodríguez, Y. P. P., Mancera Valetts, L. P., \& Sánchez Suárez, D. M. (2015). Estrategia didáctica para la formación en investigación en la educación virtual: Experiencia en la Universidad Manuela Beltrán [Didactic strategy for research training in virtual education: Experience at the Manuela Beltrán $\begin{array}{llll}\text { University]. } & \text { Revista } & \text { EAN, } & \end{array}$ https://doi.org/10.21158/01208160.n79.2015.1268

Cano Lassonde, O. M. (1970). Antecedentes internacionales y nacionales de las TIC a nivel superior: su trayectoria en Panamá [International and national background of ICT at a higher level: his trajectory in Panama]. Actualidades Investigativas En Educación, 12(3). https://doi.org/10.15517/aie.v12i3.10303

Carabelli, P. (2020). Respuesta al brote de COVID-19: tiempo de enseñanza virtual [Response to the COVID-19 Outbreak: Virtual Teaching Time Resposta ao surto COVID-19: tempo de ensino virtual]. InterCambios. Dilemas y Transiciones de La Educación Superior, 7(2), 189-198. https://n9.cl/9paxo\&tlng=es

Castillo, Y., García, N., Corredor, M., Malaver, C., \& Elizabeth, C. (2017). Evolución de la educación superior a distancia: desafíos y oportunidades para su gestión [Evolution of distance higher education: challenges and opportunities for its management]. Revista Virtual Universidad Católica Del Norte, 0(50), 81-105. https://n9.cl/xdkkr

CEPAL-UNESCO. (2020). La educacion en tiempos de la pandemia COVID-19 [Education in times of the COVID-19 pandemic]. Comisión Económica Para América Latina y El Caribe, Santiago Oficina Regional de Educación Para América Latina y El Caribe de La Organización de Las Naciones Unidas Para La Educación La Ciencia y La Cultura, 11, 11-13. https://n9.cl/b4tgl

Delgado, M y Solano, A. (2009). (2012). A standard platform for testing and comparison of mdao architectures [Una plataforma estándar para probar y comparar arquitecturas mdao]. Collection of Technical Papers AIAA/ASME/ASCE/AHS/ASC Structures, Structural Dynamics and Materials Conference. https://n9.cl/y3fv

Díaz, F. (2008). Educación y uevas Tecnologías de la información: ¿Hacia un paradigma Educativo innovador? [Education and new Information Technologies: Towards an innovative educational paradigm?]. 1-15. https://n9.cl/8m12k 


\section{CIENCIAMATRIA}

Revista Interdisciplinaria de Humanidades, Educación, Ciencia y Tecnología

Año VII. Vol. VII. N¹3. Julio - Diciembre. 2021

Hecho el depósito de ley: pp201602FA4721

ISSN-L: 2542-3029; ISSN: 2610-802X

Universidad Nacional Experimental Francisco de Miranda (UNEFM). Santa Ana de Coro. Venezuela

Marcos Adrián Luna-Rojas; Carlos Marcelo Ávila-Mediavilla

González-Cutre (2017), Martínez-Camacho, GómezRijo, y Moreno, 2010; GonzálezCutre y Moreno, 2009; Moreno et al., 2011; Moreno y González-Cutre, 2006. (2017). Estrategias didácticas y motivacionales en las clases de educación física desde la teoría de la autodeterminación [Didactic and motivational strategies in physical education classes from the theory of self-determination]. 44-62. https://n9.cl/z95ks

Jacovkis. (2011). Las TIC en América Latina: historia e impacto social [ICT in Latin America: history and social impact]. Revista Iberoamericana de Ciencia Tecnología y Sociedad, 6(18), 63-64. https://n9.cl/h4wv5

Martínez, O., Steffens, E. J., Ojeda, D. C., \& Hernández, H. G. (2018). Estrategias Pedagógicas Aplicadas a la Educación con Mediación Virtual para la Generación del Conocimiento Global [Pedagogical Strategies Applied to Education with Virtual Mediation for the Generation of Global Knowledge]. Formación Universitaria, 11(5), 11-18. https://doi.org/10.4067/s0718-50062018000500011

Mendoza Juárez, Y. L., \& Mamani Gamarra, E. J. (2012). Nacional Del Altiplano - Puno 2012. Teaching Strategies - Learning of Teachers of the Faculty of Social Sciences At the National University of the Altiplano Puno 11. https://n9.cl/1mwom

Morera-méndez, D. (1995). La motivación hacia el estudio de la Historia [The motivation to study of History]. https://n9.cl/g8ec3

Naranjo Pereira, M. L. (2009). Motivación: perspectivas teóricas y algunas consideraciones de su importancia en el ámbito educativo [Motivation: theoretical perspectives and some considerations of its importance in the educational field]. Revista Educación, 33(2), 153. https://doi.org/10.15517/revedu.v33i2.510

Palmero, F; Gómez, C; Carpi, A y Guerrero, C. (2008. (2008). Perspectiva histórica de la psicología de la motivación [Historical perspective of the psychology of motivation]. https://n9.cl/qsow9

Pando, V. (2018). Tendencias didácticas de la educación virtual: Un enfoque interpretativo [Teaching Trends in Virtual Education: An Interpretative Approach]. Propósitos y Representaciones, 6(1), 463-484. https://n9.cl/h3j0

Peña Rodríguez, F., \& Otálora Porras, N. (2018). Educación y tecnología: problemas y relaciones [Education and technology: problems and relationships]. Pedagogía y Saberes, 48, 59-70. https://doi.org/10.17227/pys.num48-7373 


\section{CIENCIAMATRIA}

Revista Interdisciplinaria de Humanidades, Educación, Ciencia y Tecnología

Año VII. Vol. VII. N¹3. Julio - Diciembre. 2021

Hecho el depósito de ley: pp201602FA4721 ISSN-L: 2542-3029; ISSN: 2610-802X

Universidad Nacional Experimental Francisco de Miranda (UNEFM). Santa Ana de Coro. Venezuela

Marcos Adrián Luna-Rojas; Carlos Marcelo Ávila-Mediavilla

Ramírez, A., Escalantes, M., \& León Salazar, A. (2008). La Educación En Tecnología: Un Reto Para La Educación [Education in Technology: a Challenge]. Educere, 731-740. https://n9.cl/pfg8h

Sánchez, O; Collazos, C y Jiménez, J. (2017). (2018). El trabajo colaborativo como estrategia didáctica para la enseñanza/aprendizaje de la programación: una revisión sistemática de la literatura [Collaborative work as a didactic strategy for teaching / learning programming: a systematic review of the litera. TecnoLógicas, 21(41), 123-7799. https://n9.cl/xal5d

Tirado, F., Santos, G., \& Tejero-Díez, D. (2013). La motivación como estrategia educativa [Motivation as an educational strategy]. Perfiles Educativos, 139, 79-92. https://n9.cl/wr5jw

Torres P., C. J. (2017). Tecnología educativa y su papel en el logrode los fines de la educación [Educational technology and its role in achieving the aims of education]. Educere, 21(68), 31-40. https://n9.cl/piiuj

Vialart Vidal, M. N. (2020). Estrategias didácticas para la virtualización del proceso enseñanza aprendizaje en tiempos de COVID-19 [TT - Didactic strategies for the virtualization of the teaching-learning process in the times of COVID-19]. Educ. Med. Super, 34(3), e2594-e2594. https://n9.cl/kj0ih 